\title{
DIGITAL SURVEY AND ALGORITHMIC MODELING IN HBIM. TOWARDS A LIBRARY OF COMPLEX CONSTRUCTION ELEMENTS.
}

\author{
V. Bagnolo ${ }^{1, *}$, R. Argiolas ${ }^{1}$, A. Cuccu ${ }^{1}$ \\ ${ }^{1}$ Department of Civil and Environmental Engineering and Architecture (DICAAR), Faculty of Engineering and Architecture, \\ Cagliari (CA), Italy - vbagnolo@unica.it
}

Commission VI, WG VI/4

KEY WORDS: Algorithmic modeling, Documentation, HBIM, Surveying, 3D modeling

\begin{abstract}
:
In the study of built heritage, the introduction of BIM models provides the advantage of a set of data that can be shared between different platforms. Despite the continuous progress in research, the modeling processes of complex construction elements, typical of historical architectures, always require a certain attention and care that involve considerable investments in terms of both resources and time. In this paper we present the first results of an ongoing research aimed at considering possible methods that can allow a simplification of the modeling processes of elements of historical architectures in the BIM environment. In particular, the research aims to explore the possibilities offered by the algorithmic modeling of complex construction elements. In the study of historical architecture, an enough recurring theme concerns those elements that can be traced back to the principles of architectural orders. In the survey of historical architecture, a quite recurring case study concerns those elements or parts of building ruled by the principles of architectural orders. One of the first elements taken into consideration was that of the column that, in its articulation in base, shaft and capital, offers three different levels of complexity of the modeling process very suitable for the research path. Starting with a collection of data acquired from digital photogrammetric modeling, we considered a initial set of different case studies that allowed us to conduct a first working hypothesis by embracing a quite wide range of possible variations starting from a basic column.
\end{abstract}

\section{INTRODUCTION}

The management of historical built heritage is distinguished by the operating complexities typical of the different phases in restoration and reuse projects, first of all the difficulty in acquiring a true knowledge of the building. The multidisciplinary approach of interventions on historical built heritage can benefit considerably from the use of Building Information Modeling (BIM). In both academic research and professional practice, Historic Building Information Modeling (HBIM) is based on mapping parametric objects onto point cloud and image survey data (Murphy et al., 2013). Although HBIM is now a well-established practice, in scientific literature the ways to create contents and libraries varies widely $\mathrm{n}$ a caseby-case basis (Pocobelli et al., 2018; Megahed, 2015; Saygi, 2013). This is due to the variety of historic architectural objects, whose complexity requires quite different approaches directly linked on each single case. This paper focuses in particular on the transition from points cloud to 3D smart model for the construction of libraries of parametric objects for historic architectural elements.

The shift from the raw 3D data to the complete and semantically enriched model often lurks the pitfalls that make the modeling process long and complex. In the shift from point cloud to BIM environment, one of the first issues is strictly linked to the practice established in the architectural survey and restoration. A second hitch is the inherent complexity of the detected elements: several features of complex historical elements can rarely find effective translation in parametric modeling without undermining the efficiency of an optimal BIM workflow.
BIM-based workflow experimented in the Cultural Heritage domain often involves a dispersion of information presenting issues related to the lack of clarity of processes. While the 3D environment requires the creation of models that allow an efficient navigation for maintaining the BIM intelligence of the objects, the field of investigation in architectural built heritage needs a good accuracy in the modeling of geometric and architectural features.

In the BIM-based modeling, sometimes the logic of standardization imposes a 3D model with generic and summary characters of the real life state. In historic contests, BIM-based modeling and data enrichment requires careful assessments on the correct level of development to be adopted in the phases of data acquisition and digital modeling (Brusaporci et al., 2018). The definition of a correct modeling methodology must consider the criteria to be adopted, objectives to be achieved and contexts of use.

BIM-based modeling can hardly support the representation of real life state as normally pursued in the survey of the historical architectural heritage. There is no question that the creation and development of rules, especially through the process of architectural survey interpretation and the introduction of principles established in architectural grammar, has to be seen as an inherently flexible, compromising and careful process, in which main rules guide the process of producing applicable and at the same time extendable models, trying to avoid the creation of general rules without making the appropriate distinctions between the different concrete situations. Starting with data collection and data processing (digital photogrammetric modeling), the procedure is aimed at identify the constitution and distribution of the different elements. Relationships and

* Corresponding author 
functional interdependence are extracted in order to identify an organic complex or a part of it: the principles of architectural orders contribute to the definition and creation of families built on the base of shape and semantics modeling. A library of profiles establishes the foundation for a critical analysis and comparison between the collected data of the architectural survey and the treatise rules. The creation of a library of parametric historic objects, aimed at minimizing the loss of reliability or data, starts from the identification and setting of the parameters useful to offer the widest possible range of variables related to each specific category of objects.

In the BIM-based workflow, the introduction of algorithmic modeling for the management of complex elements is intended to extend the model to a broader spectrum of variables responding to a wide range of real cases. Starting from the survey data, the research deals with the modeling of a single complex object that, thanks to the implementation of the algorithmic modeling, comes to define a heavy parameterization of the entire range of possible variables identified. Bim-based modeling and algorithmic modeling are developed using Revit and Dynamo platforms by Autodesk ${ }^{\circledR}$.

In order to optimize the process, it was intended to base the level of local modeling on general purpose-built families, performing the creation of semantic libraries enriching different parameters that allow us to build a knowledge-based architectural system. The goal is to expand the domain of the different cases contemplated by a single family. The presented study intends to test how it is possible to cover a wide range of real objects thanks to the algorithmic modeling without having to resort to local modeling every time.

In this paper, our study has taken into consideration the creation of a BIM object library for the column element, articulated in its functional components. The choice to start the research from this class of elements moves from reasons strictly related to the intrinsic qualities of the three functional categories that make up the column: base, shaft and capital.

The assumption that each of these three architectural elements generally needs different modeling methodologies, allows us to deal with a fairly wide range of cases. The analysis of the different declinations and variations of the column were in particular explored with reference to the shaft. While modeling the base of the column can be easily made with simple tools in the BIM environment, modeling the shaft and the capital can often be characterized by higher levels of complexity that require a greater investment of resources, making the most of the time necessary to resort to the integration of other platform programmes. Frequently, especially in relation to the typical needs of the disciplinary areas of architectural survey and restoration, the parametric modeling of the capital does not often allow a sufficiently effective parameterization, except for the simplest cases relating to the Tuscan and Doric orders. For the other three architectural orders, it is often necessary to use mesh models, especially in the most complex cases with very sophisticated decorative levels (Apollonio et al., 2012). Finally, the shaft of the column, when brought back to a simple cylinder, does not present modeling problems. But, if you want to consider the different variations in the possible solutions of the shaft, it has been seen that the creation of the shaft model can effectively make use of the algorithmic modeling. In this paper we show some case studies of complex shafts in which the application of the algorithmic modeling provides a useful solution. Algorithmic modeling offers a feasible path for creating historic objects libraries, allowing us to set up an experimental framework to shift from point clouds of complex construction elements to a 3D content model (Baik et al., 2015).

\section{STATE OF THE ART}

The most common process for modeling historical objects in BIM environment mainly follows two ways: local modeling and parametric modeling; in both cases the workflow remains almost unchanged.

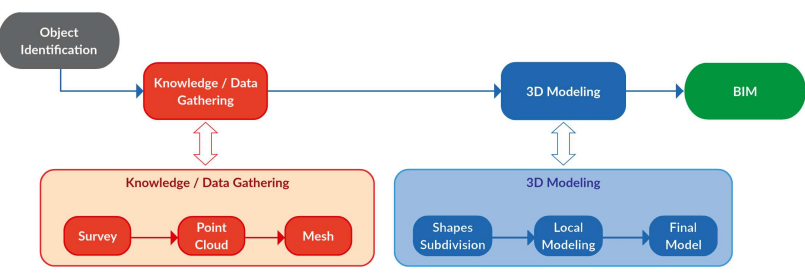

Figure 1. Classic workflow for H-BIM with external modeling tools

The first phase is the preliminary study of the object; in the case of historical objects, the analysis of the treaties is a must as a source of rules governing proportions, indispensable not only for the modeling itself of the objects but also for the formation of knowledge that will then accompany the geometric representation as well provided by the BIM methodology.

This phase, however, is not to be considered limited at the beginning of the modeling process but often continues in parallel during all the phases of study.

Once sufficient data have been collected, the object can be surveyed, thus collecting direct data on the specific case study. The practice of modeling the object under examination by photogrammetry is well established, as the potential of this method has also been analyzed several times with low-cost instruments (Chiabrando et al. 2017), especially when compared with instruments such as laser scanners, which are often put in the background from much cheaper cameras.

Results of the photogrammetry are the clouds of points (sparse or dense) and the mesh surfaces, with textures or not. From point clouds it is therefore possible to derive the measures on which to base the actual modeling; it is the modeling method that involves the first distinction of workflows, as it is possible to decide whether to use modeling tools outside the BIM environment and then import the models inside it (Figure 1) or to model them using the tools provided by the BIM software that you decide to use (Figure 2).

In the case of developing the models entirely in the BIM environment, all possible problems of data exchange and formats between the software are going to go, but often losing the advantages given by the software dedicated to modeling, such as the flexibility and complexity of the instruments (Lopez, et al., 2018).

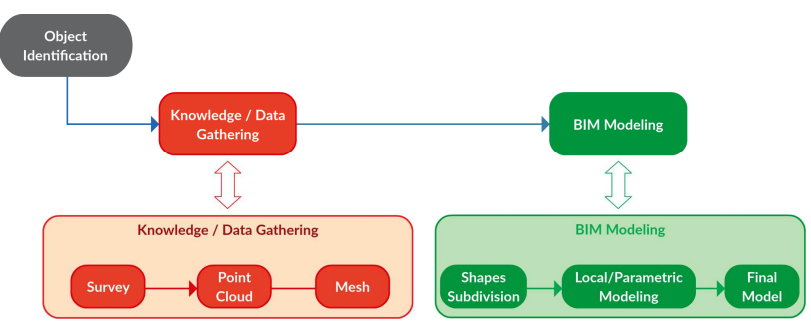

Figure 2. Workflow with internal BIM modeling tools 
As already mentioned, often in BIM we are offered two methods for object modeling, local and parametric. The local modeling consists in modeling each individual object as an element in its own right and is the most followed way in the case of non-repeated elements or with very particular characteristics; parametric modeling instead consists, against a greater complexity, in the creation of objects based on standard parameters and whose characteristics change according to them (Oreni et al., 2013; Oreni et al., 2014). Parameters can refer to practically any aspect of the object, but in our case they are limited to geometric characteristics; the family thus obtained (therefore no longer a single object) can adapt itself to a wider range of elements, provided that they share the main characteristics envisaged by the initial family.

\section{VISUAL CODING}

\subsection{Algorithmic modeling}

A possible evolution of the modeling in a bim environment is offered by the visual programming tools, nowadays more and more frequent in the various areas of programming and, as in our case, in modeling. In fact, they offer the possibility of developing algorithms that take charge of all those modeling operations necessary for the generation of $3 \mathrm{D}$ elements and repeat them every time you decide to start the routine. This entails greater flexibility in the use of modeling tools, a reduction in the work that the user will have to perform while maintaining the possibility of predicting the basic parameters on which the modeling will be performed (Figure 3). Moreover, unlike the methods previously analyzed, the operations of future implementation of new features no longer need to restart the modeling process, but often it will be sufficient to implement the new features by adding part of the code to the algorithms already developed.

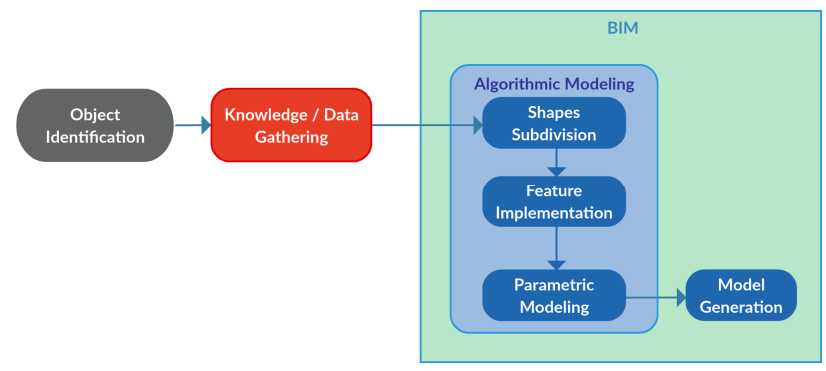

Figure 3. Algorithmic modeling

\subsection{Visual coding for data enrichment and data exchange}

Algorithmic modeling is just one of the possibilities of expansion offered by visual programming, ranging from the processing of input and output data, the modification of already modeled objects or the information connected to them (Figure 4 ), or the management of data exchange between BIM environment and other platforms (Quattrini, et al. 2018) (Figure 5), therefore, a whole new series of workflows is created that foresee within them the insertion of visual programming tools as an intermediate step or a substitute for those of classical methodologies.

The algorithmic modeling clearly shows its potentials when the objects to represent belong to macro families well defined by repeated rules in various cases; the emblematic case is the columns. In fact, they present, even if in countless configurations, elements or characteristics common to many cases, either for shapes, for geometric rules or for rhythms; this is precisely what allow to codify the columns according to the classical orders, and the method we followed in our study does not differ from that of the treatises, namely the search for geometric and compositional rules, and how they can generate the various existing configurations.

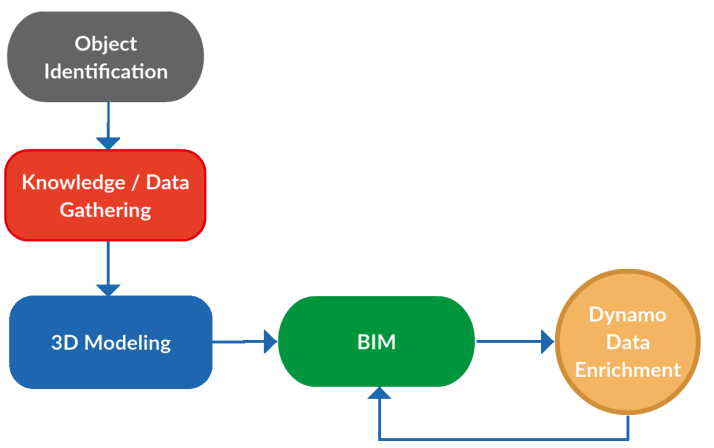

Figure 4. Visual Coding for Data Enrichment

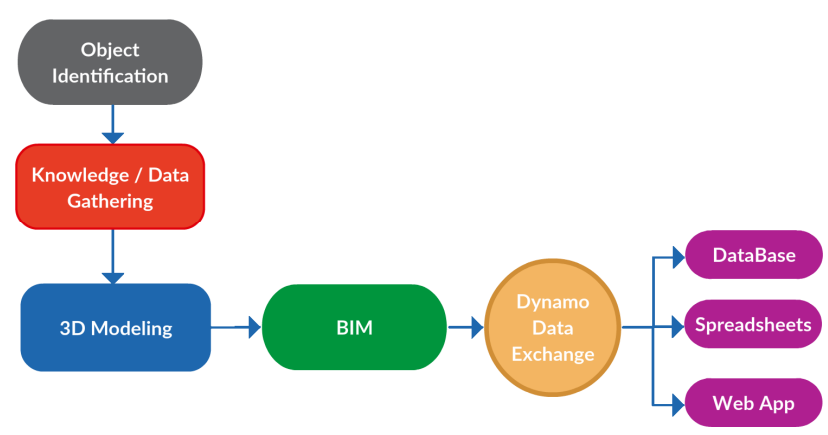

Figure 5. Visual Coding for Data Exchange

\subsection{First algorithm development: Doric column with entasis}

The survey of a Doric column with a smooth-surfaced shaft, is the first case study considered. The element chosen for the survey is one of the columns in the façade of the 19th century Civil Hospital in Cagliari, by the architect Gaetano Cima. The choice was dictated by the simplicity of the elements, typical of the Doric order; considering the shaft, it can be easily associated with the primitive of the cylinder.

A feature that distinguishes the shaft of the Doric column from the cylinder is the presence of the entasis, which can be implemented in the object thanks to the algorithmic modeling; if we look for the study of the entasis in the treaties, for example in those of Vignola, we see that its construction is analyzed as a series of instructions that gradually define the profile of the column (Barozzi Da Vignola, 1602). The height of the column shaft and the diameter at its imoscapo constitute the parameters of the model. By varying the geometry of the profiles, all the variants (curved profiles and polygonal profiles) can be obtained. The reference rule is that of the architectural orders codified by Vignola. This choice does not exclude the possibility of resorting to other treaties if the theoretical model does not correspond to the specific one found. The geometric construction is an algorithm of instructions that allows us to determine a series of profiles.

These instructions can be easily executed in Dynamo (Figure 6) and are handled exactly like an algorithm: we have starting data 
(diameters at the imoscapo, at the entasis and at the top of the column, height of the entasis and height of the column), we have a series of instructions and finally we have a final "object", the column profile. As we can see from the image, in dynamo the organization is the same:

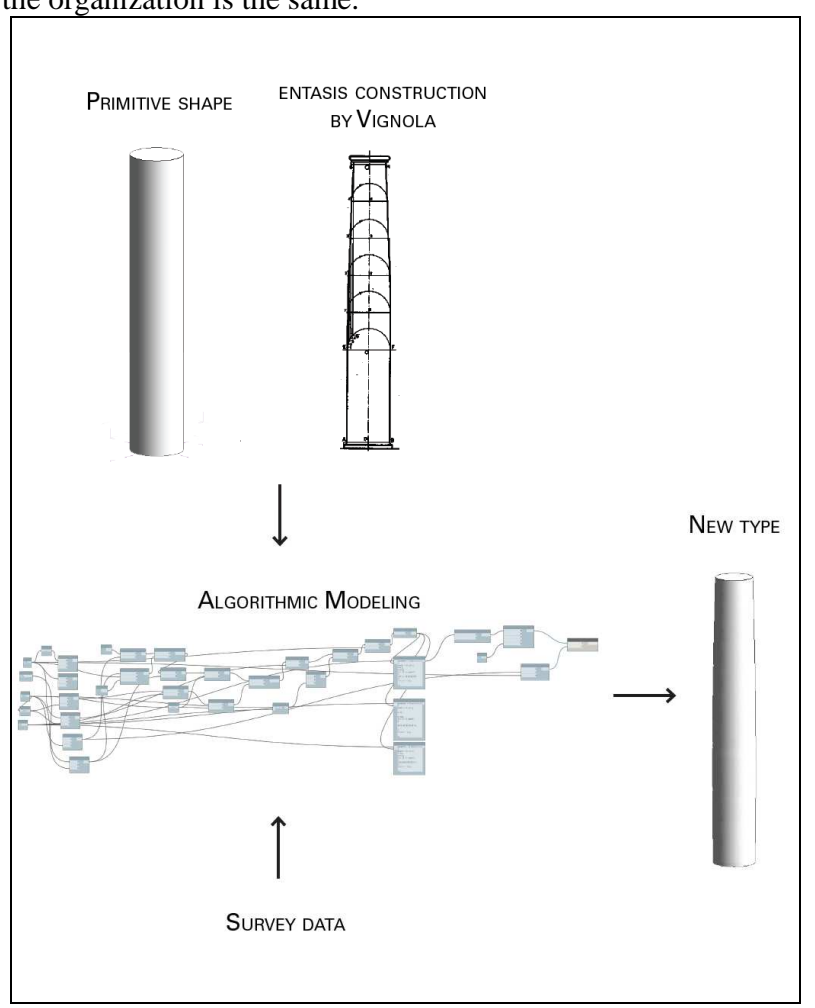

Figure 6. Development of entasis algorithm

Once the algorithm for generating the entasis has been defined, starting from the initial parameters entered, it can generate any column with a smooth and entasised shaft that follows the constructive rules illustrated by Vignola. This also means that the future user who needs to model a shaft of this type is relieved of all design and modeling operations, but will have only the burden of introducing the initial parameters coming from the survey.

\subsection{Implementation workflow}

3.4.1 Palazzo Belgrano. A second implementation of the shaft object is achieved by considering a banded column. The evolution of the model requires an algorithm able to generate a banded shaft. Further specific characters, such as the number of column drums or the fluted shaft, further evolve the model. The final model was then tested including case studies and development of data acquisition and digital modeling of selected columns. The case study is that of the columns in the entrance hall of Palazzo Belgrano in Cagliari. The columns are composed by a smooth shaft with an entasis, punctuated by a succession of bands with a circular profile. A further algorithmic modeling is necessary in order to include the "definition" for the banded shaft. The implementation does not involve rewriting the algorithm from scratch, but it will be inserted as a new series of commands able to create new features (Figure 7).

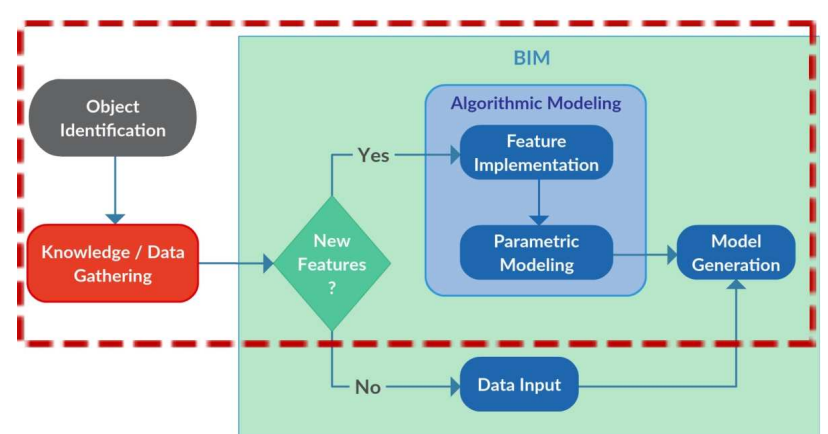

Figure 7. Visual Coding for features implementation

The modeling is quite easy, being the bands simple cylinders defined by diameter and height; their positioning is as simple as the interaxis between the bands is constant. It is therefore necessary to create a new part of the algorithm that will have as input data the dimensions of the bands, diameter and height of the notes, their number and the distance between the two bands; from these data a band is generated and will then be copied in a vertical series along the shaft already modeled by the algorithm. The result (Figure 8) is a new algorithm capable of generating any smooth shaft with entasis, and whether or not there are smooth and sequential cylindrical bands.

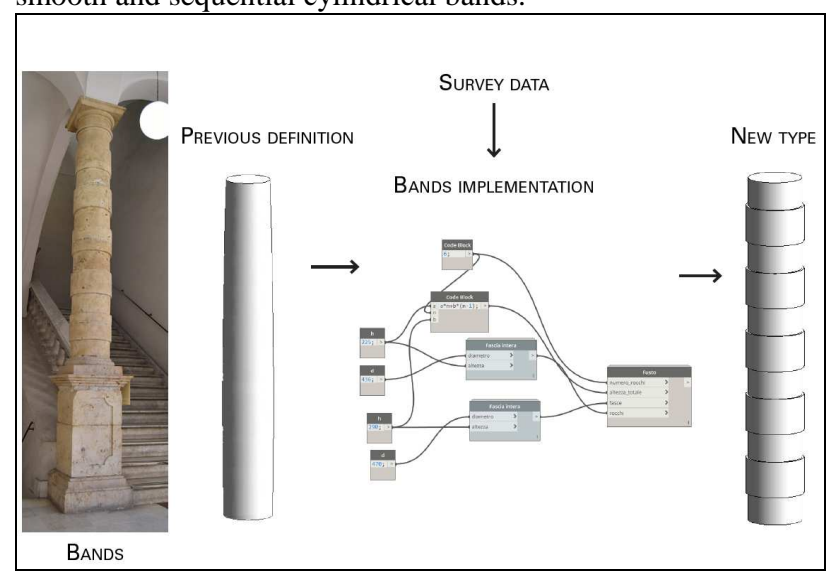

Figure 8. Banded type implementation

Villa in Via dei Genovesi. Not far from Palazzo Belgrano we find, in via dei genovesi, a private villa whose entrance is defined by a portal with columns.

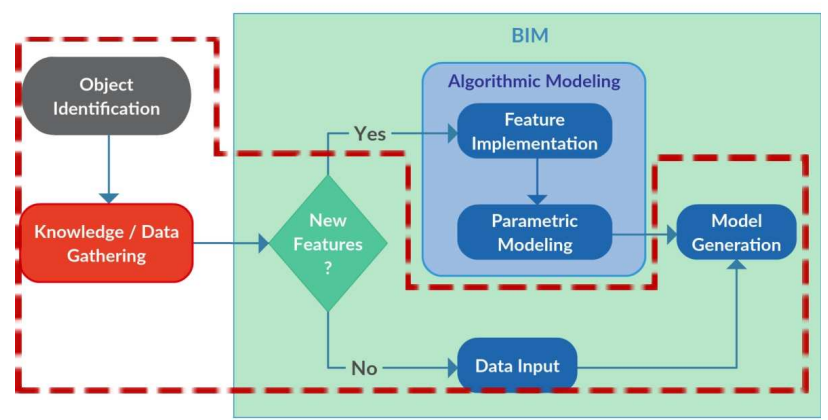

Figure 9. Different model with already developed algorithm 
These columns have a smooth shaft and cylindrical bands also smooth, thus falling perfectly into the type of shafts just treated (Figure 9). After a first phase of survey from which to derive the initial parameters, their modeling is almost immediate: you set the starting data, start the dynamo algorithm and in a few moments the model is generated (Figure 10).

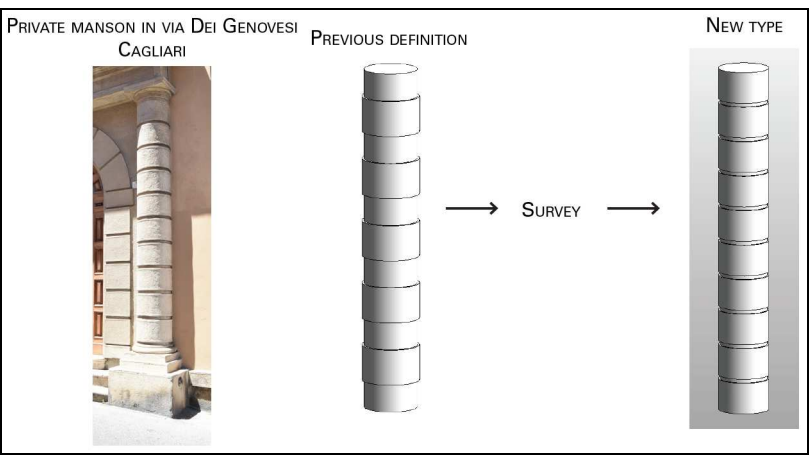

Figure 10. Application of the "Banded type" algorithm

3.4.2 Royal Saltworks, Arc-et-Senans, Doubs. A new feature that can be implemented is found in the house of the director of the royal saltworks in Doubs. The columns of the entrance have a smooth shaft with bands, but in this case the bands have a square profile. It is therefore necessary to implement the possibility of modeling square bands, or more generally polygonal; it is a question of maintaining the same workflow but adding the possibility of defining a new polygonal profile with parameters such as the number of sides and their measurement (Figure 11). Being the shape of the bands the only difference with the previous type, the number and the position of the bands is easily managed as in the other cases examined.

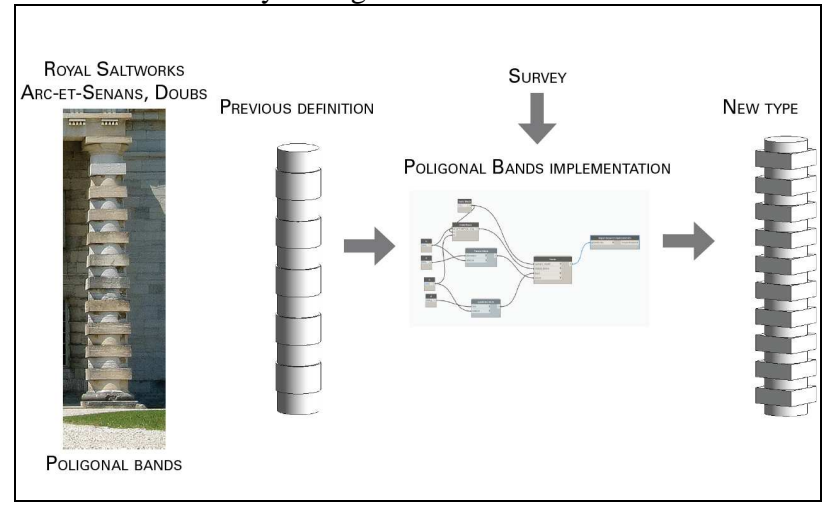

Figure 11. Polygonal bands implementation

3.4.3 Saint-Étienne-du-Mont Church, Paris. Another typological variation is found in the columns of the facade of the church of S. Etienne. The columns have a fluted shaft and decorated bands; leaving aside the question of decorated bands that will require a more detailed future reasoning, we have focused on the processing of the fluted shaft, a very common feature. The implementation in this case concerned the possibility of defining a grooved profile to be extruded, as an alternative to the initial circular profile. The necessary data are, in addition to the already present diameter and height, the number of grooves and their measurements; once the fluted shaft has been implemented (Figure 12), it is therefore possible to model types with fluted shafts without bands or with circular or polygonal bands.

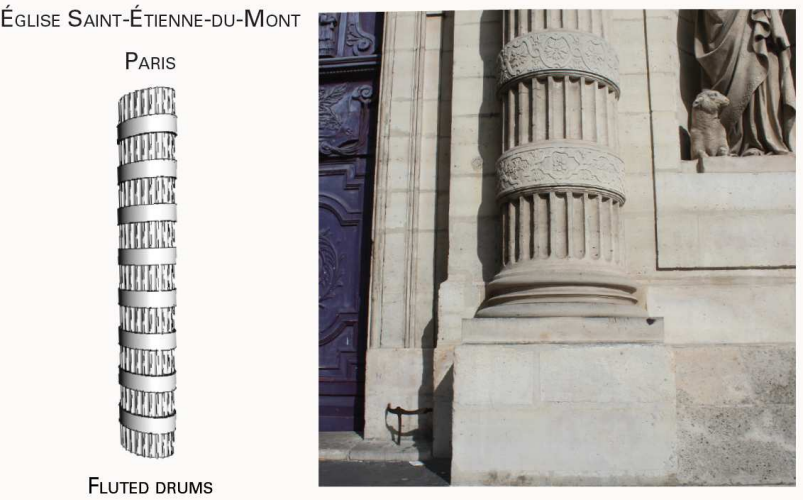

Figure 12. Implementation of fluted drums

3.4.4 Mauritian complex of Santa Croce. Another case study that we present is that of the columns of the atrium of the Santa Croce complex in Cagliari. The columns have a fluted shaft with circular bands; the bands, however, are articulated in an alternation of three parts, two with a larger diameter and the central one with a smaller diameter. It is possible to imagine the bands thus composed as an overlap of three bands, all having the same profile but different diameters, superimposed on the basis of their height; the "sub-bands" so imagined can easily be generated by the algorithm that generates the circular bands, the implementation therefore concerns the creation of a macro band given precisely by the combination of sub-bands. In order to make the field of use wider, it was decided to leave the number of bands to alternate free and to fix as the only limitation, modifiable in the future, that the sequence is given by the two measures that alternate in an odd series (first and last element of the same type).

This new type of band can be combined with any type of shaft already analyzed giving life to a new series of cases. Moreover, if we implement the change of profile from circular to polygonal as seen in the case of Doubs, we can obtain shaft, both smooth and grooved, and multiple bands of polygonal shape. These implementations allow us to represent the angled columns and the pilasters still present in the atrium of S.Croce, having a shaft with a square fluted section and multiple strips always with a square profile (Figure 13).

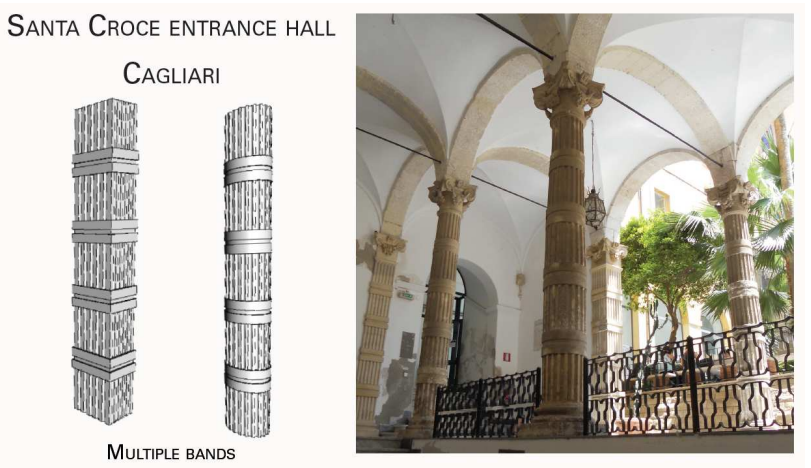

Figure 13. Multi-bands and polygonal drums application in Santa Croce complex (Cagliari) 
3.4.5 Palazzo Brondo - Zapata, Cagliari. The last configuration taken into consideration in this paper is the one found in the entrance portal of the Palazzo Brondo-Zappata in Cagliari. The columns of the portal have a smooth circular shaft with square bands; the peculiarity is the irregularity in the arrangement of the bands.

In this case the approach has been to divide the sequence of bands into full and empty and to represent it through a string of 0 and 1 where for every 1 the algorithm goes to insert a band defined as in the previous cases.

This method sets as a basic condition that the bands are equal to each other and that the sequence can be divided into "modules" of constant dimensions.

The algorithm thus defined therefore needs as a new parameter the string that defines the sequence in which to place the bands, for example 110111011, the rest of the algorithm remains unchanged (Figure 14).
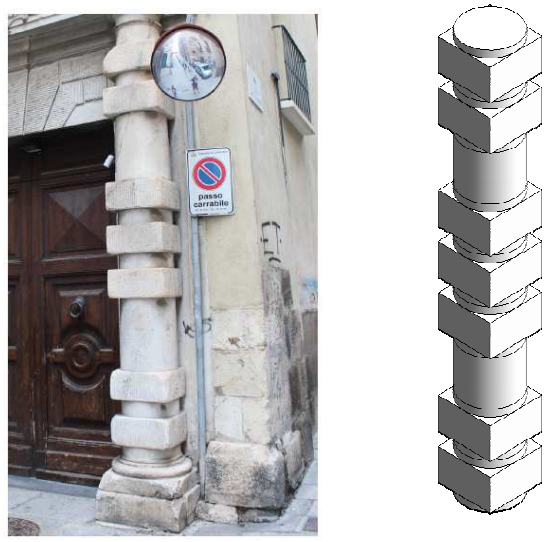

Figure 14. Irregular bands sequence by strings in Palazzo Brondo (Cagliari)

3.4.6 Palazzo delle Scienze, Cagliari. A variant of what we saw for the Palazzo Brondo-Zappata is that of the columns of the Palazzo delle Scienze in Cagliari, where there are only two square bands at the base of the shaft. By setting a string like 11000000 , the requested model is obtained (Figure 15).
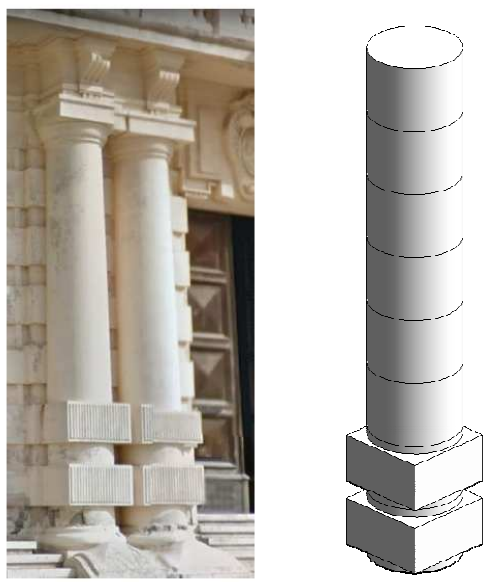

Figure 15. Application of the string based dislocation in Palazzo delle Scienze (Cagliari)
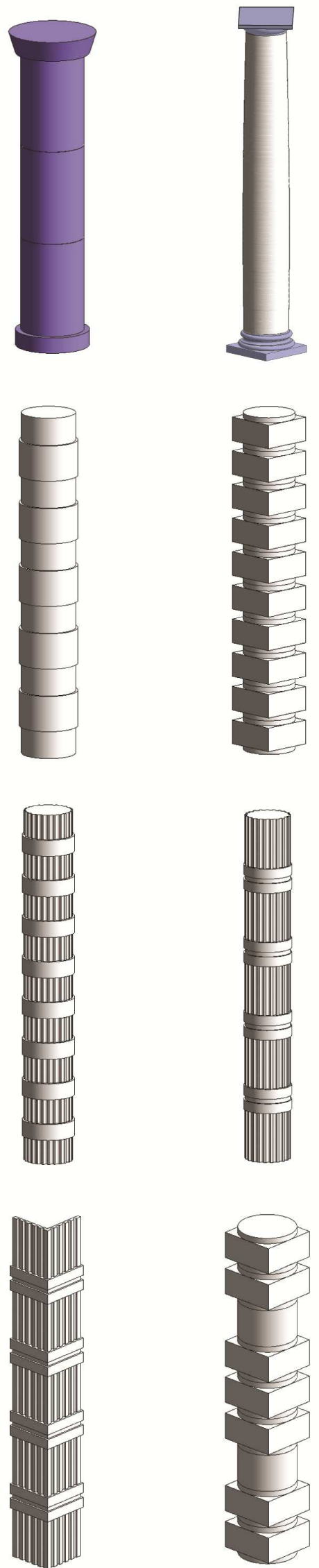

Figure 16. comparison between a standard Autodesk Revit ${ }^{\circledR}$ column (in purple) and the models obtainable from the developed algorithm (greyscale) 


\section{RESULTS}

The study up to now has led us to the development of a family of shafts capable of representing a great variety of types, although the cases treated are quite limited (Figure 16). Moreover, in proceeding with the analysis of the various types, a remarkable modeling speed was found in those cases in which there was no need to impose new features, practically eliminating the direct modeling operations by the user. In fact, once the initial parameters from the survey are obtained, the generation of the model is practically instantaneous, without the need to act manually with the 3D modeling tools.

Moreover, in spite of the obvious approximations necessary for a "series" modeling, as regards the representation, the objects obtained have a good correspondence with the real models; this correspondence will then be evaluated from time to time according to the purpose of the modeling, and possibly evaluate to what extent it is convenient to go with the precision.

The method adopted so far has proved to be reliable in the various cases treated, so that in most cases the difficulties of application are attributable to aspects more related to mere programming rather than to the methodology.

As mentioned earlier in this paper, the choice of columns as an object of study was also dictated by the variety of configurations in which this architectural element can be presented; this variety also makes the implementation work that can be carried out hypothetically infinite. It is certainly necessary to expand the cases treated to deal with increasingly complex types, and to understand to what extent the adopted method remains robust when the columns treated present considerable complexity. Examples can be the management of twin columns or with particular decorations.

It should also be noted that the study so far focused on the shafts of the columns, but the same process can also be applied to the base and to the capital of the columns; if it is true that in most cases the bases present a degree of difficulty of representation comparable to that of the shaft, the capital deserves instead a more targeted reasoning as even the simpler types have more complex elements to be schematized. It will therefore be necessary to evaluate within which limits of detail it is convenient to proceed with the algorithmic modeling and when it is better to give the step to a mesh model, perhaps obtained from photogrammetry.

Finally, another aspect worth investigating is the interface with which the user can interact with the algorithms when inserting parameters; indeed, it has been evident from the first examples treated how the system of modules offered natively by computational BIM design software Dynamo for the insertion of data is very thin and often not very intuitive.

It is also true that the integration of the Python language within Dynamo offers valid design tools for any graphical interfaces that can improve the end user experience.

\section{REFERENCES}

Apollonio F., Gaiani M., and Zheng S, 2012. BIM-Based MOdeling and Data Enrichment of Classical Architectural Building. In: Sciret-It, Vol 3 Issue 2, pp. 41-62.

Barozzi Da Vignola, G.., 1602. Regola delli cinque ordini d'architettura di M. Iacomo Barozzio da Vignola. In Roma apresso Giovani Orlandi.

Baik A, Yaagoubi R., and Boehm J., 2015. Integration of Jeddah historical BIM and 3D GIS for documentation and restoration of historical monument. In: International Archives of the Photogrammetry, Remote Sensing and Spatial Information Sciences, 40(5)/W7(5W7), pp. 29-34.

Brusaporci, S., Maiezza, P., and Tata, A. 2018. A framework for architectural heritage HBIM semantization and development. In: ISPRS - International Archives of the Photogrammetry, Remote Sensing and Spatial Information Sciences, Vol. XLII-2, pp.179-184.

Chiabrando, F., Lo Turco, M., and Santagati, C., 2017. Digital invasions: from point clouds to historical building object modeling (H-BOM) of a UNESCO WHL site. In: ISPRS International Archives of the Photogrammetry, Remote Sensing and Spatial Information Sciences, Vol. XLII-2/W3, pp.171178.

López, F., Lerones, P., Llamas, J., Gómez-García-Bermejo, J., and Zalama, E., 2018. A Review of Heritage Building Information Modeling (H-BIM). In: Multimodal Technologies and Interaction, 2(2), p.21.

Megahed, N., 2015. Towards a Theoretical Framework for HBIM Approach in Historic Preservation and Management. In: International Journal of Architectural Research: ArchNet-IJAR, 9(3), p.130.

Murphy, M., McGovern, E., and Pavia, S., 2013. Historic Building Information Modelling - Adding intelligence to laser and image based surveys of European classical architecture. In: ISPRS Journal of Photogrammetry and Remote Sensing, 76, pp.89-102.

Oreni D., Brumana R., Georgopoulos A., and Cuca B., 2013. HBIM for conservation and management of built heritage: towards a library of vaults and wooden bean floors. In: ISPRS Annals of the Photogrammetry, Remote Sensing and Spatial Information Sciences, Volume II-5/W1, 2013.

Oreni, D., Brumana, R., Georgopoulos, A., and Cuca, B., 2014. HBIM Library Objects for Conservation and Management of Built Heritage. In: International Journal of Heritage in the Digital Era, 3(2), pp.321-334.

Pocobelli, D., Boehm, J., Bryan, P., Still, J., and Grau-Bové, J., 2018. BIM for heritage science: a review. In: Heritage Science, $6(1)$.

Quattrini, R., Battini, C., and Mammoli, R., 2018. HBIM to VR. Semantic awareness and data enrichment interoperability for parametric libraries of historical architecture. In: ISPRS International Archives of the Photogrammetry, Remote Sensing and Spatial Information Sciences, XLII-2, pp.937-943.

Saygi, G., and Remondino, F., 2013. Management of Architectural Heritage Information in BIM and GIS: State-ofthe-Art and Future Perspectives. In: International Journal of Heritage in the Digital Era, 2(4), pp.695-713.

Revised December 2018 